\title{
ANALYTIC STUDY OF RATIONAL QUINTIC SURFACES HAVING NO MULTIPLE CURVES
}

\author{
BY H. N. HUBBS
}

1. Introduction. The purpose of this paper is to derive the equations of certain of the rational quintic surfaces without multiple curves discussed synthetically by Montesano.* The equations of the surfaces are found by applying Cremona transformations to certain well known rational surfaces of order three or four.

2. Surface of Order Five with Four Triple Points. This surface is the transform by the cubic transformation $T_{t e t} \dagger$ of a general cubic surface $\phi_{3}$ through the vertices of the tetrahedron. The equation of the surface is

$$
\begin{aligned}
\phi_{5} \equiv & y_{1}^{2}\left[y_{2}^{2} u+y_{3}^{2} u^{\prime}+y_{4}^{2} u^{\prime \prime}+A y_{2} y_{3} y_{4}\right] \\
& +y_{1}\left[y_{4}^{2} \phi_{2}+y_{2} y_{3}\left(B y_{3} y_{4}+y_{2} u^{\prime \prime \prime}\right)\right] \\
& +y_{2} y_{3} y_{4}\left[C y_{3} y_{4}+D y_{2} y_{4}+E y_{2} y_{3}\right]=0,
\end{aligned}
$$

where $u, u^{\prime}, u^{\prime \prime}, u^{\prime \prime \prime}$ are linear in $\left(y_{3}, y_{4}\right),\left(y_{2}, y_{4}\right),\left(y_{2}, y_{3}\right),\left(y_{3}, y_{4}\right)$, respectively, and $\phi_{2}$ is quadratic in $\left(y_{2}, y_{3}\right)$, and where $A, B, C, D$ and $E$ are constants. The points whose coordinates are $(1,0,0,0)$, $(0,1,0,0),(0,0,1,0),(0,0,0,1)$ are triple points, with non-composite tangent cones at each of the points.

3. The Surface $\phi_{5}$ with Three Ordinary Triple Points and a Tacnode. The surface $\phi_{5}$ is the transform by $T_{t e t}$ of a quartic surface with a double conic passing through three of the vertices of the tetrahedron and having the fourth vertex at a general point of the surface. The section of $\phi_{5}$ by a plane through the tacnode and two triple points is a straight line and a pair of conics passing through these points.

The equation of $\phi_{4}$ with a double conic is

$$
\begin{aligned}
\phi_{4} \equiv\left[\sum a_{i} x_{j} x_{k}\right]^{2}-4 x_{4}^{2}\left[\psi_{2}+x_{4} \psi_{1}\right]=0, \\
(i, j, k=1,2,3,4, \cdots, i \neq j \neq k),
\end{aligned}
$$

* Montesano, Napoli Rendiconti, (3), vol. 7 (1901), pp. 67-106.

$\dagger$ Hudson, Cremona Transformations in Plane and Space, Cambridge University Press, 1927, pp. 301-303. 
where $\psi_{1}$ and $\psi_{2}$ are linear and quadratic, respectively, in the variables $\left(x_{1}, x_{2}, x_{3}\right)$. The surface $\psi_{5}$ is

$$
\phi_{5} \equiv y_{4}^{3} \phi_{1}^{2}-4 y_{4} \psi_{4}-4 y_{1} y_{2} y_{3} \phi_{2}=0,
$$

where $\phi_{1}$ is linear in $\left(y_{1}, y_{2}, y_{3}\right), \psi_{4}$ is quadratic, and $\phi_{2}$ linear in $\left(y_{2} y_{3}, y_{1} y_{3}, y_{1} y_{2}\right)$. The point $(0,0,0,1)$ is a tacnode on $\phi_{5}$, and $(0,0,1,0),(0,1,0,0),(1,0,0,0)$ are triple points with noncomposite tangent cones.

4. The Surface $\phi_{5}$ with Three Ordinary Triple Points and a Tacnode, the Tacnode lying with one of the Triple Points on a Line of $\phi_{5}$ Situated in the Tangent Plane at the Tacnode. This surface is the transform by $T_{t e t}$ of a rational surface $\phi_{4}$ of order four, having a double line and two double points in a plane through the double line. One fundamental point of the transformation is on the double line, two at the double points, and the fourth a general point of $\phi_{4}$. The plane of the double points and double line is tangent to $\phi_{4}$ along the line joining the double points. The transform of $\phi_{4}$ by $T_{t e t}$ is

$$
\phi_{5} \equiv\left(y_{2}-y_{1}\right)^{2} y_{4}{ }^{3}+\left(y_{2}-y_{1}\right)^{2} y_{4} \psi_{2}+y_{4} \psi_{4}+\psi_{5}=0,
$$

where $\psi_{2}, \psi_{4}$, and $\psi_{5}$ are forms in $\left(y_{1}, y_{2}, y_{3}\right)$ of the order of their subscripts. The triple points are then $(1,0,0,0),(0,1,0,0)$, $(0,0,1,0)$, and the tacnode is $(0,0,0,1)$.

5. The Surface $\phi_{5}$ with Two Triple Points and Two Tacnodes. A space Cremona transformation of order three is defined by the web of surfaces of order three passing through three fixed conics $k_{2}, k_{2}^{\prime}, k_{2}^{\prime \prime}$ which lie in distinct planes, have one point in common, and meet by pairs in three points. The conjugate system is defined by cubic surfaces having in common three nonconcurrent coplanar lines and a space cubic curve meeting each line once.

The inverse of this transformation carries a quadric surface through one of the lines and the intersection of the other two into a surface $\psi_{5}$ of order five having two of the fundamental conics as double curves and the third a simple curve. The transformation $T_{t e t}$ with fundamental points at the intersections of these conics carries $\psi_{5}$ into a surface $\phi_{5}$ which has two triple points and two tacnodes. The tacnodes are the images of the double conics of $\psi_{5}$. 
The equation $\phi_{5}$ obtained by the above procedure is

$$
\begin{aligned}
\phi_{5} \equiv & y_{4}^{2}\left[y_{2} y_{3} u_{1}+A y_{1} y_{3} u_{2}+B y_{1} y_{2} u_{3}+C y_{1} y_{2} y_{3}\right. \\
& \left.+u_{1}\left(D y_{3} u_{2}+D y_{2} u_{3}+E y_{2} y_{3}\right)\right] \\
& +y_{4}\left[y_{2} y_{3} u_{4}+A y_{1} y_{3} u_{5}+B y_{1} y_{2} u_{6}+u_{1}\left(F y_{3} u_{5}+D y_{2} u_{6}\right)\right. \\
& \left.+u_{4}\left(F y_{3} u_{2}+D y_{2} u_{3}+E y_{2} y_{3}\right)\right]+u_{4} u_{5} u_{6}=0,
\end{aligned}
$$

where

$$
\begin{array}{ll}
u_{1} \equiv b_{4} y_{2}+\frac{b_{3} c_{4}}{c_{2}} y_{3}, & u_{4} \equiv y_{2}\left(b_{1} y_{1}+b_{3} y_{3}\right)+\frac{b_{3} c_{1}}{c_{2}} y_{1} y_{3}, \\
u_{2} \equiv a_{4} y_{1}+\frac{a_{3} c_{4}}{c_{1}} y_{3}, & u_{5} \equiv y_{1}\left(a_{2} y_{2}+a_{3} y_{3}\right)+\frac{a_{3} c_{2}}{c_{1}} y_{2} y_{3}, \\
u_{3} \equiv a_{4} y_{1}+\frac{a_{2} b_{4}}{b_{1}} y_{2}, & u_{6} \equiv y_{1}\left(a_{2} y_{2}+a_{3} y_{3}\right)+\frac{a_{2} b_{3}}{b_{1}} y_{2} y_{3}
\end{array}
$$

and the double conics of the first transformation are

$$
\begin{aligned}
& x_{1}=0, \quad \sum a_{i} x_{j} x_{k}=0, \quad(i, j, k=2,3,4, \cdots, i \neq j \neq k), \\
& x_{2}=0, \quad \sum b_{i} x_{j} x_{k}=0, \quad(i, j, k=1,3,4, \cdots, i \neq j \neq k), \\
& x_{3}=0, \quad \sum c_{i} x_{j} x_{k}=0, \quad(i, j, k=1,2,4, \cdots, i \neq j \neq k) .
\end{aligned}
$$

The points $(0,1,0,0)$ and $(0,0,1,0)$ are tacnodes and the points $(1,0,0,0),(0,0,0,1)$ are triple points. The line $y_{2}=0, y_{3}=0$ lies on the surface.

6. The Surface $\phi_{5}$ with One Triple Point and Three Tacnodes. The inverse of the first transformation of $\$ 5$ carries a quadric surface through the intersections of the fundamental straight lines into a surface $\psi_{6}$ of order six having the fundamental conics as double curves, their common intersection a four-fold point, and the three points of intersection of these conics by pairs three-fold points. The transformation $T_{t e t}$, with fundamental points at these multiple points, carries $\psi_{6}$ into a $\phi_{5}$ with one triple point and three tacnodes. The procedure described above gives

$$
\begin{aligned}
\phi_{5} \equiv & y_{4}^{2}\left[y_{1} y_{2} y_{3}+u_{1}\left(y_{3} u_{2}+y_{2} u_{3}+y_{2} y_{3}\right)\right. \\
& \left.+y_{1} u_{2}\left(u_{3}+y_{3}\right)+y_{1} y_{2} u_{3}\right]+y_{4}\left[u_{1}\left(y_{3} u_{5}+y_{2} u_{6}\right)\right. \\
& +u_{4}\left(y_{3} u_{2}+y_{2} u_{3}+y_{2} y_{3}\right)+y_{1} u_{2} u_{6}+y_{1} u_{5}\left(u_{3}+y_{3}\right) \\
& \left.+y_{1} y_{2} u_{6}\right]+u_{4}\left(y_{3} u_{5}+y_{2} u_{6}\right)+y_{1} u_{5} u_{6}=0
\end{aligned}
$$


where $u_{i}$ are as indicated in $\S 5$. The point $(0,0,0,1)$ is an ordinary triple point and the points $(1,0,0,0),(0,1,0,0)$, $(0,0,1,0)$ are tacnodes. The tangent cones at the tacnodes are, respectively,

$$
\begin{gathered}
\left(a_{2} y_{2}+a_{3} y_{3}+a_{4} y_{4}\right)^{2}=0, \quad\left(b_{1} y_{1}+b_{3} y_{3}+b_{4} y_{4}\right)^{2}=0, \\
\left(c_{1} y_{1}+c_{2} y_{2}+c_{4} y_{4}\right)^{2}=0 .
\end{gathered}
$$

7. The Surface $\phi_{5}$ with an Ordinary Triple Point and a Tacnodal Triple Point. A Cremona transformation is defined by the web of quadric surfaces containing a fixed conic and an arbitrary point. A special case of this transformation arises when the point is on the fixed conic.

If the conic of this web is tangent to a rational quartic surface with a double line at a general point of the line, the transformation defined by the web carries the quartic surface into a quintic having an ordinary triple point and a triple point with an adjacent infinitesimal double line, ${ }^{*}$ or tacnodal triple point, both lying on the fundamental conic.

The equation of the quintic obtained by the above transformation is

$$
\begin{gathered}
\phi_{5} \equiv y_{4}^{2} y_{3}^{2} \psi_{1}\left(y_{1}, y_{2}\right)+y_{4} y_{3} \phi_{1}\left(y_{1}, y_{2}, y_{3}\right) \cdot \phi_{2}\left(y_{1}, y_{2}\right)+y_{3}^{2} y_{1} \psi_{2}\left(y_{1}, y_{2}\right) \\
+y_{3} \psi_{4}\left(y_{1}, y_{2}\right)+\psi_{5}\left(y_{1}, y_{2}\right)=0 .
\end{gathered}
$$

The point $(0,0,1,0)$ is an ordinary triple point and $(0,0,0,1)$ a tacnodal triple point. In the plane $y_{3}=0$ are five straight lines on the surface, images of the residual intersections of the fundamental conic and the quartic surface. On $\phi_{5}$ are eighteen conics passing through the triple points and lying by pairs in nine planes through the triple points.

8. The Surface $\phi_{5}$ with One Tacnodal Triple Point and One Tacnode. In the following the vertices of the tetrahedron of reference are the points $A_{i}$, those of the conjugate system $B_{i}$, $(i=1,2,3,4)$.

A rational surface of order four of the third type of Noether has a double point $A_{4}$ which is a cusp in a general plane section through it. The surface has a simple line passing through the double point; a general section through this line is a cubic curve

* Segre, Annali di Matematica, (2), vol. 25 (1896), pp. 1-53. 
having the line as inflectional tangent at $A_{4}$. The tangent cone at $A_{4}$ is the plane $p$ taken twice; this is tangent to $\phi_{4}$ along the line. A section by this plane is a conic tangent to the line at $A_{4}$.

In the transformation of $\$ 7$, let the conic be tangent to $\phi_{4}$ at $A_{4} . \quad T \equiv\left[F_{2} \equiv C_{2} A_{4} p, F_{2}^{\prime} \equiv C_{2}^{\prime} B_{4} p^{\prime}\right]$. Under $T, \phi_{4} \sim \phi_{5}:: B_{3}^{2} B_{4}^{3}$, where $B_{3}$ is a tacnode, and $B_{4}$ is a tacnodal triple point. The conic $C_{2}^{\prime}$ is on $\phi_{5}$. As in (7), the point $B_{4}$ is a tacnodal triple point, the tangent planes being $p^{\prime}$ taken twice, and $p_{1}^{\prime}$ containing $C_{2}^{\prime}$.

The equation of the quintic surface obtained by this method is

$$
\begin{aligned}
\phi_{5} \equiv & y_{1} y_{3}^{2} y_{4}^{2}+2 y_{3} y_{4}\left[y_{1} y_{3} \phi_{1}+\phi_{3}-y_{1}\left(y_{1} y_{2}+y_{1}^{2}+y_{2}^{2}\right)\right] \\
& +\left(y_{1} y_{2}+y_{1}^{2}+y_{2}^{2}\right)\left[y_{1}\left(y_{1} y_{2}+y_{1}^{2}+y_{2}^{2}\right)-2\left(y_{1} y_{3} \phi_{1}+\phi_{3}\right)\right] \\
& -y_{1}^{2} y_{3}^{3}+y_{1} y_{3}^{2} C_{2}+y_{1} y_{3} C_{3}+y_{1} C_{4}=0,
\end{aligned}
$$

where $\phi_{1}, \phi_{3}$, and $C_{i}$ are forms in $\left(y_{1}, y_{2}\right)$ of the order of their subscripts.

The point $(0,0,0,1)$ is a tacnodal triple point and $(0,0,1,0)$ is a tacnode lying on a line of $\phi_{5}$ situated in the simple tangent plane at the triple point. The section of $\phi_{5}$ by the simple tangent plane at the triple point is the conic $y_{3} y_{4}-y_{2}^{2}=0$ and the line $y_{2}$ $=0$ counted three times; this line is the image of the simple line of $\phi_{4}$.

9. The Surface $\phi_{5}$ with an Ordinary Triple Point and an Oscnode. A quadratic Cremona transformation is defined by the quadric surfaces $F_{2}$ having in common two generators and osculating at their point of intersection.* Let the generators be $l_{1}$ and $l_{2}$ and their point of intersection $A_{1}$. The transformation is

$$
T \equiv\left[F_{2}:: l_{1}, l_{2}, A_{1}, F_{2}^{\prime} \equiv l_{1}^{\prime}, l_{2}^{\prime}, B_{1}\right] \text {. }
$$

Let $A_{1}$ be a generic point on a monoidal quartic surface $\phi_{4}$, and let $l_{1}, l_{2}$ each osculate $\phi_{4}$ at $A_{1}$. Let the triple point of $\phi_{4}$ be $A_{4}$. Under $T, \phi_{4} \sim \phi_{5}$ with an ordinary triple point at $B_{4}$ and an oscnode at $B_{1}$.

A general straight line through $A_{1}$ has three residual intersections with $\phi_{4}$; hence the image straight line has three intersections with $\phi_{5}$ not at $B_{1}$. A general straight line meets $\phi_{4}$ in

*Hudson, loc. cit., pp. 197-198. 
four points; hence its image conic has four points in common with $\phi_{5}$ not at $B_{1}$; that is, at $B_{1}$ are three consecutive double points on the image conic or $B_{1}$ is an oscnode on $\phi_{5}$. The equation of $\phi_{4}$ is

$$
\phi_{4} \equiv \psi_{3} x_{4}+\psi_{4}=0
$$

where

$$
\begin{aligned}
& \psi_{3} \equiv A x_{1}^{3}+w_{1} x_{1}^{2}+w_{2} x_{1}+w_{3}, \\
& \psi_{4} \equiv B x_{1}^{2} x_{2} x_{3}+u_{3} x_{1}+u_{4},
\end{aligned}
$$

and where $w_{i}, u_{i}$ are binary forms in $\left(x_{2}, x_{3}\right)$ of order $i$. The line $x_{2}=0, x_{4}=0$ osculates $\phi_{4}$ at $(1,0,0,0)$. The equations of transformation are

$$
\rho x_{1}=B\left(y_{1} y_{4}-y_{2} y_{3}\right), \rho x_{2}=A y_{2} y_{4}, \rho x_{3}=y_{3} y_{4}, \rho x_{4}=y_{4}^{2} .
$$

The equation of the resulting quintic is

$$
\begin{aligned}
& B^{2}\left(y_{1} y_{4}-y_{2} y_{3}\right)^{2}\left[A B y_{1}+w_{1}\right] \\
&+B\left(y_{1} y_{4}-y_{2} y_{3}\right)\left[w_{2} y_{4}+u_{3}\right]+y_{4}\left[w_{3} y_{4}+u_{4}\right]=0,
\end{aligned}
$$

where $w_{i}, u_{i}$, are the above forms in $\left(y_{2}, y_{3}\right)$. The point $(0,0,0,1)$ is a triple point and $(1,0,0,0)$ is an oscnode.

10. The Surface $\phi_{5}$ with an Oscnode and a Tacnode. Applying the transformation of $\S 9$ to a quartic surface of the first type of Noether, we find

$$
\begin{aligned}
& \phi_{5}=B^{2}\left(y_{1} y_{4}-y_{2} y_{3}\right)^{2} {\left[A B y_{1}+y_{4}+u_{1}\right] } \\
&+B\left(y_{1} y_{4}-y_{2} y_{3}\right)\left[u_{2} y_{4}+u_{3}\right]+u_{4} y_{4}=0,
\end{aligned}
$$

where $u_{i}$ are binary forms in the variables $\left(y_{2}, y_{3}\right)$ of order $i$. The point $(0,0,0,1)$ is a tacnode and $(1,0,0,0)$ an oscnode.

11. The Surface $\phi_{5}$ with an Oscnode and a Double Point of the First Order. Such a surface is the transform by the above transformation of a quartic of the second type of Noether. The equation of this quintic is

$$
\begin{aligned}
\phi_{5} \equiv & \phi_{1}^{2} y_{4}^{3}+2 y_{4}^{2}\left[y_{3} \phi_{1}\left(y_{3}+\psi_{1}\right)+\phi_{3}\right] \\
& +y_{4}\left\{y_{3}^{4}+2 y_{3}^{3} \psi_{1}-\left[D \psi_{1}+F \phi_{1}+D\left(2 F y_{1}+G y_{2}\right)\right.\right. \\
& \left.\left.+B K_{1} y_{1}+K_{2} y_{2}\right] y_{2} y_{3}^{2}+y_{2} y_{3} \phi_{2}+y_{2}^{3} \phi_{1}^{\prime}\right\} \\
& +y_{2} y_{3} \psi_{3}+A B^{3} y_{1} y_{2}^{2} y_{3}^{2}=0,
\end{aligned}
$$


where

$$
D=b_{1} B, \quad E=b_{2} A, \quad F=c_{1} B, \quad G=c_{2} A, \quad \phi_{1}=D y_{1}+E y_{2},
$$

and where

$$
\begin{aligned}
& \psi_{1}=F y_{1}+(G-D) y_{2}, \\
& \phi_{3}=A B^{3} y_{1}^{3}+B^{2} K_{3} y_{1}^{2} y_{2}+B K_{4} y_{1} y_{2}^{2}+K_{5} y_{2}{ }^{3}, \\
& \phi_{2}=-2 A B^{3} y_{1}^{2}+B\left(K_{6}-2 B K\right) y_{1} y_{2}-B K_{4} y_{2}{ }^{2}, \\
& \phi_{1}^{\prime}=B K_{7} y_{1}+\left(K_{8}-B K_{4}\right) y_{3} \\
& \psi_{3}=\left(2 D F-B K_{1}\right) y_{2} y_{3}^{2}+B\left(B K_{3}-K_{6}\right) y_{2}{ }^{2} y_{3}-2 F y_{3}{ }^{3}-B K_{7} y_{2}{ }^{3} .
\end{aligned}
$$

The point $(1,0,0,0)$ is an oscnode, and $(0,0,0,1)$ is a double point of the first order.

12. The Surface $\phi_{5}$ with an Oscnode and a Double Point of the Second Order. Such a surface is the transform of a quartic surface of the third type of Noether. The equation of this quintic is

$$
\begin{aligned}
\phi_{5} \equiv & B^{2}\left(y_{1} y_{4}-y_{2} y_{3}\right)^{2} \phi_{1}+B\left(y_{1} y_{4}-y_{2} y_{3}\right)\left[\phi_{3}+y_{2} y_{4} \psi_{1}\right] \\
& +y_{2}^{2} y_{4}\left[A y_{2} y_{4}+\phi_{2}\right]=0,
\end{aligned}
$$

where $\phi_{1}$ is linear in all variables, and where $\psi_{1}, \phi_{2}, \phi_{3}$ are forms in $\left(y_{2}, y_{3}\right)$ of the order of their subscripts. The point $(1,0,0,0)$ is an oscnode, and $(0,0,0,1)$ is a double point of the second order.

13. The Surface $\phi_{5}$ as a General Member of a Homaloidal Family of a Cremona Space Transformation. The quintic surfaces discussed above are all rational and their $(1,1)$ representations on a plane $\pi$ are known. Cremona* has shown that the set of space transformations having $\phi$ as a general member of the first homaloidal family corresponds to a set of plane Cremona transformations in $\pi$.

If, by a Cremona transformation, $\phi_{n}$ is the transform of a rational $\psi_{m}$ which is a general member of the homaloidal families of $k$ Cremona transformations, then $\phi_{n}$ will serve as the general member of the homaloidal families of $k$ Cremona transformations.

* Cremona, Istituto Lombardo Rendiconti, (2), vol. 4 (1871), pp. 269-279; and Annali di Matematica, (2), vol. 5 (1871), pp. 131-162. 
14. Conclusion. Each of the rational quintic surfaces discussed above can serve as a general member of the homaloidal family of a Cremona transformation. The surfaces of $\$ \$ 5,6,9$ are the transforms, by Cremona transformations, of a general quadric surface. The surface of $\$ 2$ is the transform of a general $\phi_{3}$; that of $\$ 10$ is the transform of a quartic surface of the first type of Noether which is the transform of a general $\phi_{3}$. The surfaces of $\$ \S 8,12$ are the transforms of a quartic surface of the third type of Noether which is the transform of a special quartic of the first type of Noether with a double point in the tangent plane at the tacnode; this plane is tangent to the surface along the line joining the double points, ${ }^{*}$ and this surface is a transform of a general $\phi_{3}$. The surfaces of $\S \S 7,11$ are transforms of a quartic with a double line. $\dagger$ The surface of $\$ 4$ is the transform of a quartic with a double line and two double points coplanar with the double line; the homaloidal family of which this $\phi_{4}$ is a general member have in common the double line and also have contact along the line joining the double points. The surface of $\S 3$ is the transform of a quartic with a double conic. $\ddagger$

Cornell University

* Noether, Mathematische Annalen, (3), vol. 33 (1889), pp. 546-571.

$\dagger$ Montesano, Roma Rendiconti, (4), vol. 5-2 (1889), pp. 123-130.

$\ddagger$ Aroldi, Giornale di Matematiche, (3), vol. 11 (1920), pp. 175-192. 